\title{
Spherical mode analysis of planar frequency-independent multi-arm antennas based on its surface current distribution
}

\author{
G. Armbrecht, O. Klemp, and H. Eul \\ Universität Hannover, Institut für Hochfrequenztechnik und Funksysteme, Hannover, Germany
}

\begin{abstract}
Deployment in the design of mobile radio terminals focuses on the implementation of multiradio transmission systems, using a multiplicity of different radio standards combined with high-speed data communication over multiple-input multiple-output (MIMO) and multimode diversity techniques. Hence, planar log.-per. four-arm antennas are predistined to meet the requirements of future mobile multiradio RF-frontends and will be introduced and analysed in terms of an efficient spherical mode analysis by means of surface current distribution in order to derive an analytic access to MIMO- and polarisation-diversity performance computation. A remarkable parameter reduction and a faster numerical analysis with respect to conventional techniques may be achieved. The sources in the near-field antenna region are based on the numerical computation of surface currents involving the finite element method (FEM). Relations between the variations of the geometrical antenna parameters and the excitation of discrete spherical modes are presented and will be analysed in detail.
\end{abstract}

\section{Introduction}

This article focuses on the in-depth analysis of planar multiarm antennas for the application in future mobile broadband communication environments. For the practical realisation of multiradio terminals which combine the reception of a multiplicity of different communication standards like cellular phone (e.g. GSM, WCDMA, UMTS), wireless networks (e.g. WLAN, HIPERLAN, WiMAX), proximity (e.g. Bluetooth, RFID, UWB) and broadcasting services (e.g. FM, DVB-H) in one box, antennas with a compact footprint and almost frequency-independent electromagnetic antenna transmission behaviour are required. Furthermore the char-

Correspondence to: G. Armbrecht

(armbrecht@hft.uni-hannover.de) acteristics of multipath propagation by independently fading broadband and orthogonal transmission channels are exploited for the increased demand of high-speed data transmission. Following Jensen and Wallace (2004) this may lead to the application of MIMO and multimode diversity techniques in the future standardisation of novel communication systems. In this case especially planar log.-per. four-arm antennas in a frequency range from 1.0 to $6.0 \mathrm{GHz}$ with selfcomplementary antenna shapes may contribute to an excellent RF-frontend performance, due to the orthogonality between the two linear excitation modes of the antenna that results in a high degree of cross-polarisation suppression and marginal envelope correlation behaviour (see Klemp et al., 2005). Such a multi-arm antenna performance is mandatory for the use in future mobile MIMO and polarisation diversity applications.

Hence, the efficient analysis based on a spherical mode expansion (SME) is applied to planar log.-per. multi-arm antennas and especially evaluated in case of four-arm antennas with a trapezoidal geometry, as proposed in Klemp and Eul (2005). The theory of spherical mode expansion first investigated by Stratton (1941) may be effectively applied to solve the scalar, homogeneous Helmholtz-PDE, as shown extensively e.g. in Werner and Mittra (2000) into a series of well-known spherical eigenfunctions. In the past, many authors reported about the successful application to a multiplicity of wave propagation problems, e.g. Cassegrainian-fed paraboloids (see Potter, 1967) and the investigation of multiple scattering of electromagnetic waves (see Bruning and Lo, 1971). Even for todays techniques of spherical antenna near field measurement this series expansion is a fundamental basis, as described by Ludwig (1971). In Chen and Simpson (1991) and Chen et al. (1992) this kind of spatial field approach is expanded to a straight-forward spherical series representation by means of the antenna surface current distribution and was applied to an infinitely thin dipole structure. The main advantage of this field approach is the opportu-

Published by Copernicus GmbH on behalf of the URSI Landesausschuss in der Bundesrepublik Deutschland e.V. 


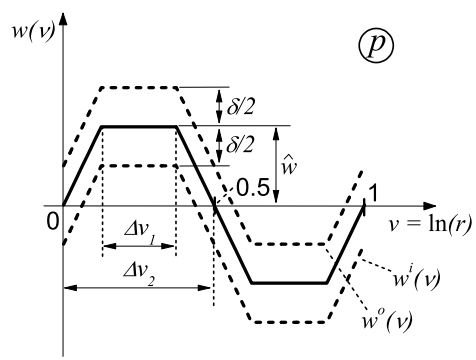

a) original plane

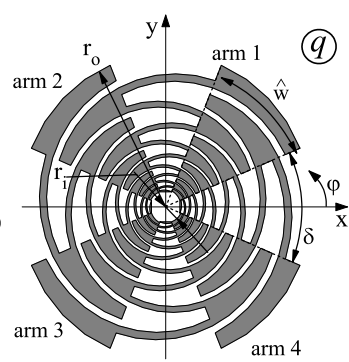

b) image plane
Fig. 1. Trapezoidal antenna geometry in the original (p) and image plane (q).

nity of separating the given source-region from the requested far-field region which leads to an efficient parameter reduction and a faster numerical analysis with respect to conventional computational techniques. The article is organized as follows: A short introduction into the generatation methology of planar log.-per. antennas will be given in Sect. 2. In Sect. 3.1 and 3.2 the details of the applied SME field approach are presented, in which the sources in the antenna near-field region are obtained from the surface current distribution derived from a commercial field-simulator based on the finite element method (FEM).

In consequence of the coordinate-transform between the source- and the far-field region a new, modulated eigenmode spectrum will be introduced in Sect. 3.3. Furthermore, we will highlight the spatial- and frequency-based dependence of the spherical eigenmode excitation for this special kind of frequency-independent log.-per. planar antennas in Sect. 3.4. A verification of the spherical field approach is accomplished in Sect. 4 involving a conventional, moment-based field calculation method in the special case of planar antennas. We will investigate the convergence properties of the series expansion with respect to the underlying coordinate system. Finally, Sect. 5 derives a relationship between the geometrical properties of log.-per. four-arm antennas of different geometrical shapes and the distinct spectra of spherical eigenmodes.

\section{Log.-per. multi-arm antenna design}

In this section the planar logarithmically-periodic selfcomplementary trapezoidal antenna designed for a use in the frequency range of 1.0 to $6.0 \mathrm{GHz}$ is introduced that serves as an initial point for the spherical eigenmode analysis. In Klemp et al. (2005) this kind of antenna structure was identified as an optimum in terms of cross-polarization decoupling, cut-off frequency and footprint. Such characteristics in combination with an almost frequency-independent inputimpedance and radiation pattern behaviour above the cutoff frequency are favorable for the application in multiradio transmission systems. One of the specialized designs is a

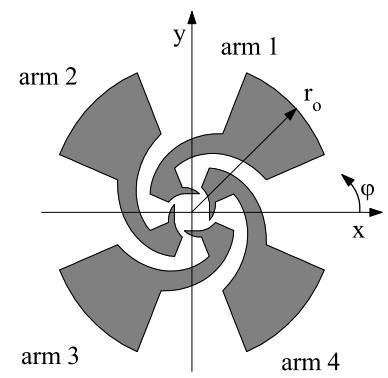

a) trapezoid antenna, $M=1$

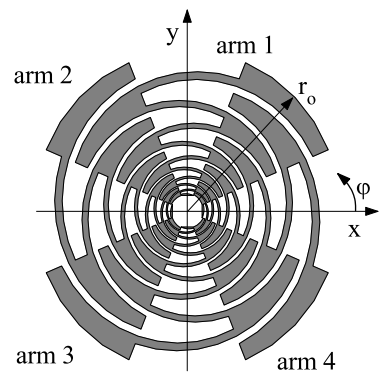

b) trapezoid antenna, $M=5$
Fig. 2. Trapezoidal antenna geometry in the image plane $q$ with $M=1$ and $M=5$ basis function periods.

Table 1. Typical geometry settings of planar trapezoidal log.-per. antenna structures

\begin{tabular}{lccccccc}
\hline parameter & $r_{i} / \mathrm{mm}$ & $r_{o} / \mathrm{mm}$ & $N_{A}$ & $M$ & $\hat{w} /{ }^{\circ}$ & $s_{f l} / \%$ & $\delta /{ }^{\circ}$ \\
\hline value & 5.0 & 50.0 & 4 & 5 & 45 & 50 & 45 \\
\hline
\end{tabular}

four-arm $\left(N_{A}=4\right)$ trapezoidal antenna, which provides two operational modes with dual-linear orthogonal polarisation fed by odd-phase port excitation of each pair of two opposite arms. The properties of dual-polarisation may be used in order to enhance channel capacity in MIMO transmission system or reduce signal fading due to polarisation diversity reception.

Referring to Fig. 1, the log.-per. antenna geometry is generated using Eq. (1) for the inner $r^{i}$ and outer $r^{o}$ limitation radii in the image plane $q$, as follows:

$$
\left\{\begin{array}{l}
r^{i}(\varphi) \\
r^{o}(\varphi)
\end{array}\right\}=e^{v} \cdot \exp \left\{\begin{array}{l}
j w^{i}(v) \\
j w^{o}(v)
\end{array}\right\}
$$

In Eq. (1), $w^{i}$ and $w^{o}$ denote the inner and outer basis function in the original plane $p$, as depicted in Fig. 1a. A trapezoidal basis geometry is defined in terms of multiple parameter settings. A typical set of these parameters is shown in Table 1, where $N_{A}$ denotes the number of antenna arms and $M$ the number of periods of the trapezoidal basis function in the $p$-plane. Additional parameters are the angular spread $\hat{w}$, the slope of the trapezoidal flanks $s_{f l}$ and the arm width $\delta$, where $\delta=45^{\circ}$ is used for self-complementary geometries. As given in Klemp et al. (2005), the inner and outer radii determine the upper and the lower antenna cut-off frequencies, respectively.

In this paper the distinct trapezoidal antenna geometries with $M=1$ and $M=5$ periods and cut-off frequencies at approximately $f_{c}^{M_{1}} \approx 4.2 \mathrm{GHz}$ and $f_{c}^{M_{5}} \approx 1.6 \mathrm{GHz}$ are extensively evaluated. To get a profound insight of these structures, they are depicted in Fig. 2. 


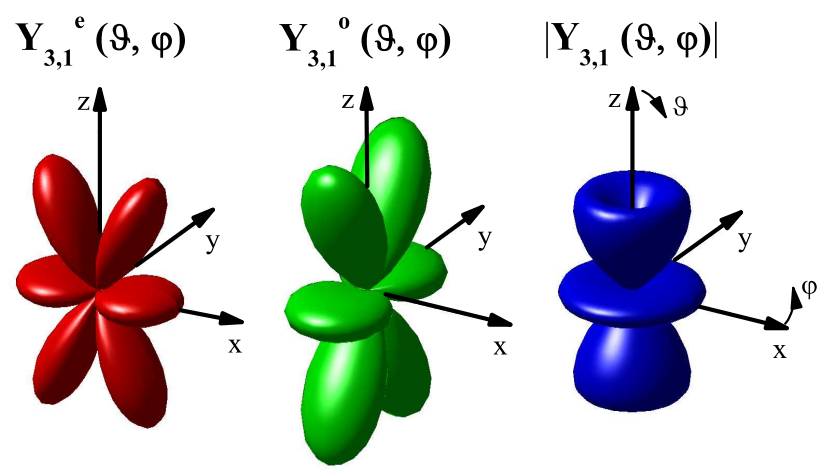

Fig. 3. Spherical harmonics $Y_{3,1}(\vartheta, \varphi)$ subdivided into even, odd and magnitude spectrum.

\section{Spherical mode expansion (SME)}

According to Werner and Mittra (2000) the spherical mode expansion is a systematic field approximation technique for arbitrary source-free regions in linear and partial homogeneous materials. The results possess a rather mathematical than physical character and need to be interpreted in terms of coordinate plane dependency. In this article the spherical multipole expression is utilized to characterize the radiation behaviour of planar log.-per. antennas introducing a novel modal description. In this section a brief introduction of the chosen, straight-forward field approach will be given.

\subsection{Modal solutions of scalar Helmholtz equation}

Any radiation and field propagation problem in ordinary spherical coordinates exhibits a solution to the scalar, homogeneous Helmholtz partial differential equation (PDE) for any functional dependency $\Psi(\boldsymbol{r})$, given by:

$\Delta \underline{\Psi}(\boldsymbol{r})+k^{2} \underline{\Psi}(\boldsymbol{r})=0$.

Solving the Eq. (2) in terms of coordinate separation techniques into a radial and a transversal functional dependency leads to a double sum representation of orthogonal spherical eigenfunctions $Y_{\mathrm{n}, \mathrm{m}}(\vartheta, \varphi)$ with integer eigenvalues $n$ and $m$ describing the degree and the order of the spherical harmonics (see Stratton, 1941), as follows:

$\underline{\Psi}(\boldsymbol{r})=\sum_{n} \sum_{m} \underline{c}_{\mathrm{n}}^{\mathrm{m}} \cdot z_{\mathrm{n}}^{\mathrm{m}}(k r) \cdot Y_{\mathrm{n}, \mathrm{m}}(\vartheta, \varphi)$.

In Eq. (3) $\underline{c}_{\mathrm{n}}^{\mathrm{m}}$ is a complex weighting factor and $z_{\mathrm{n}}^{\mathrm{m}}$ denotes a spherical cylinder function.

In detail the Eq. (4) expresses the composition of the spherical eigenfunctions subdivided into even (e) and odd (o) modes which are given by orthogonal order-dependent trigonometric dependency (periodic Lamé products) of the azimuth angle $\varphi$.

$$
\left\{\begin{array}{l}
Y_{\mathrm{n}, \mathrm{m}}^{\mathrm{e}}(\vartheta, \varphi) \\
Y_{\mathrm{n}, \mathrm{m}}^{\mathrm{o}}(\vartheta, \varphi)
\end{array}\right\}=N_{\mathrm{n}}^{\mathrm{m}} \cdot P_{\mathrm{n}}^{\mathrm{m}}(\cos \vartheta) \cdot\left\{\begin{array}{c}
\cos (m \varphi) \\
\sin (m \varphi)
\end{array}\right\}
$$

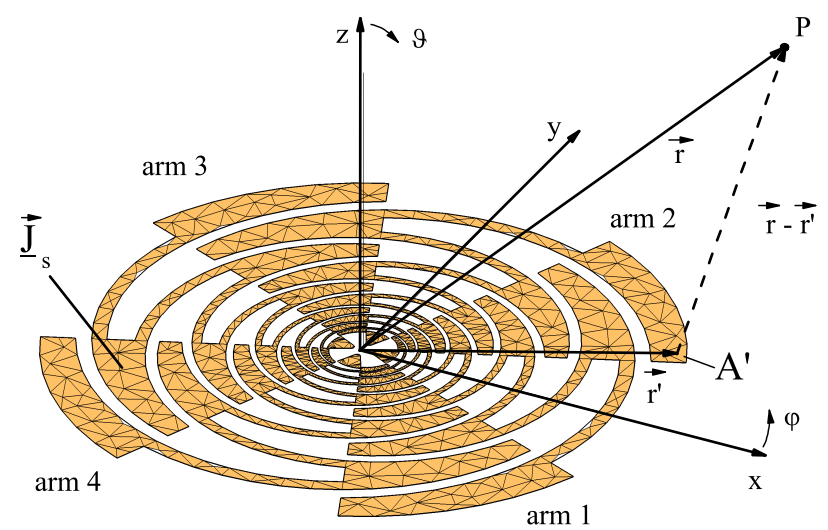

Fig. 4. Trapezoidal antenna $(M=5)$ in spherical coordinates with a given electric current distribution $\underline{\boldsymbol{J}}_{s}\left(\boldsymbol{r}^{\prime}\right)$ affecting the magnetic vector potential $\underline{\boldsymbol{A}}(\boldsymbol{r})$ in the requested far-field point $P$.

Furthermore $P_{\mathrm{n}}^{\mathrm{m}}$ denotes the elevation dependency in terms of associated Legendre polynomials as non-periodic Lamé products. $N_{\mathrm{n}}^{\mathrm{m}}$ represents a normalisation coefficient in accordance to Werner and Mittra (2000). Both quantities depend on the degree $n$ and order $m$ of the spherical harmonics, respectively.

Figure 3 depicts the spatial magnitude distribution in terms of the even- and odd-parts as well as the magnitude plot of the spherical eigenfunction $Y_{3,1}(\vartheta, \varphi)$ of the degree $n=3$ and the order $m=1$.

\subsection{Current-based field approach}

In order to determine the magnetic vector potential $\underline{\boldsymbol{A}}(\boldsymbol{r})$ in any requested field point, we will refer to a planar region $A^{\prime}$ that exhibits a source distribution of electric surface currents $\underline{\boldsymbol{J}}_{s}\left(\boldsymbol{r}^{\prime}\right)$, as shown in Fig. 4. According to Eq. (5), the magnetic vector potential $\underline{\boldsymbol{A}}(\boldsymbol{r})$ may be computed involving the Kirchhoff integral of a given surface current distribution $\underline{J}_{s}\left(\boldsymbol{r}^{\prime}\right)$ that causes free-space radiation represented by the Green's free-space function $G\left(\boldsymbol{r}, \boldsymbol{r}^{\prime}\right)=e^{-j k\left|\boldsymbol{r}-\boldsymbol{r}^{\prime}\right|} /\left|\boldsymbol{r}-\boldsymbol{r}^{\prime}\right|$.

$\underline{\boldsymbol{A}}(\boldsymbol{r})=\frac{1}{4 \pi} \iint_{\left(A^{\prime}\right)} \underline{\boldsymbol{J}}_{s}\left(\boldsymbol{r}^{\prime}\right) \cdot \frac{e^{-j k\left|\boldsymbol{r}-\boldsymbol{r}^{\prime}\right|}}{\left|\boldsymbol{r}-\boldsymbol{r}^{\prime}\right|} d A^{\prime}$.

Therefore, the Green's free space function for homogeneous, source-free regions is a solution of the Helmholtz-PDE (2) and may be expanded into a spherical eigenmode series, as given in Sect. (3). The spherical wave expansion corresponds directly to Klemp and Eul (2005) and will therefore not be demonstrated in detail.

Magnetic current distributions $\underline{\boldsymbol{J}}_{m}$ may also be analysed in the same manner leading to the electric vector potential $\underline{\boldsymbol{F}}$. An enhancement to magnetic currents is not required, because planar log.-per. antenna structures may be completely 

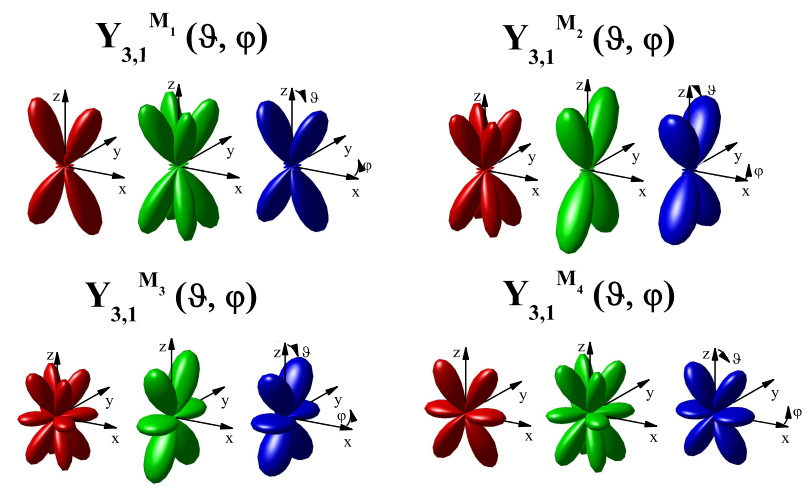

Fig. 5. Modulated spherical harmonics spectrum $Y_{3,1}(\vartheta, \varphi)$.

described in terms of real electric surface currents $\underline{\boldsymbol{J}}_{s}$.

$$
\begin{aligned}
\underline{\boldsymbol{A}}(\boldsymbol{r})=\frac{e^{-j k r}}{r} \sum_{n=0}^{\infty} \sum_{m=0}^{n} j^{\mathrm{n}}\left[\underline{\boldsymbol{a}}_{\mathrm{n}}^{\mathrm{m}} \cdot Y_{\mathrm{n}, \mathrm{m}}^{\mathrm{e}}(\vartheta, \varphi)\right. \\
\left.+\underline{\boldsymbol{b}}_{\mathrm{n}}^{\mathrm{m}} \cdot Y_{\mathrm{n}, \mathrm{m}}^{\mathrm{o}}(\vartheta, \varphi)\right] .
\end{aligned}
$$

For that reason Eq. (6) provides a compact spherical eigenfield approach for $\underline{\boldsymbol{A}}(\boldsymbol{r})$ that is characterized by a complete coordinate separation between near- and far-field quantities adhering to the Fraunhofer approximation. The current sources in the near-field region given by the surface current distribution $\underline{\boldsymbol{J}}_{s}$ of the planar antenna described by the complex vector coefficients $\underline{\boldsymbol{a}}_{\mathrm{n}}^{\mathrm{m}}$ and $\underline{\boldsymbol{b}}_{\mathrm{n}}^{\mathrm{m}}$ of the field expansion. The Green's function accounts for the spherical wave propagation of the magnetic vector potential, whereas the remaining terms incorporate its spatial profile.

For a numerical evaluation of $\underline{\boldsymbol{A}}(\boldsymbol{r})$ given by Eq. (6), the limitation of the series expansion to finite SME degrees $N$ will be essential leading to impairments in the approximated far-field representation. For that purpose Ludwig (1971), Narasimhan et al. (1985) and Chen and Simpson (1991) introduced a SME convergence criteria to reduce the field impairments significantly, if the SME degree $N$ is derived for $N \geq k a$, whereas $k$ denotes the scalar wavenumber of the free-space and $a$ represents the radius of the sphere including all current sources in the near-field. In our special case of planar trapezoidal antennas the radius $a$ correlates with the maximum radius $r_{\mathrm{O}}=50 \mathrm{~mm}$ of the antenna footprint.

\subsection{Modulated eigenmode spectra}

To accomplish the numerical antenna analysis based on the antenna's surface currents, we applied a conventional FEM field-simulator in order to obtain the correct near-field source distribution on the antenna surface. For further analysis the far-field representation of the antenna's radiation characteristics is required, which leads to the application of the far-field approximation by Fraunhofer. By this means, a simple relationship between the SME expanded magnetic vector potential $\underline{\boldsymbol{A}}(\boldsymbol{r})$ according to Eq. (6) and the resulting electric field components $E_{\vartheta}$ and $E_{\varphi}$ in spherical coordinates is exhibited, given by,

$\boldsymbol{E}(\vartheta, \varphi)=\left[\begin{array}{l}E_{\vartheta} \\ E_{\varphi}\end{array}\right]=-j k \eta\left[\begin{array}{l}A_{\vartheta} \\ A_{\varphi}\end{array}\right]$,

where $\eta=120 \pi \Omega$ denotes the value of the characteristic free-space impedance. The field approximation given by Eq. (7) is only applicable in spherical coordinates $(r, \vartheta, \varphi)$ and neglects the radial field component dependency. A certain coordinate transform between cartesian and spherical coordinates has to be taken into account, which may be expressed by four metric coefficients $M_{i}(\vartheta, \varphi)$, as follows:

$$
\begin{aligned}
M_{1}(\vartheta, \varphi) & =\cos (\vartheta) \cos (\varphi) ; & M_{2}(\vartheta, \varphi) & =\cos (\vartheta) \sin (\varphi) \\
M_{3}(\varphi) & =-\sin (\varphi) ; & M_{4}(\varphi) & =\cos (\varphi) .
\end{aligned}
$$

The metric coefficients $M_{i}(\vartheta, \varphi)$, with $i=1, \ldots, 4$, represent the transform of cartesian components $\left\{A_{x}, A_{y}\right\}$ of the magnetic vector potential to the spherical components $\left\{A_{\vartheta}, A_{\varphi}\right\}$, which leads to a new set of orthogonal spatial eigenfunctions. The new set of eigenfunctions $Y_{\mathrm{n}, \mathrm{m}}^{\mathrm{M}_{\mathrm{i}}}$ may be represented in terms of the original set of spherical harmonics, as in Eq. (4), using the coefficients $M_{i}(\vartheta, \varphi)$, as given in Eq. (8). This leads to a quadruple of modified spherical harmonics $Y_{\mathrm{n}, \mathrm{m}}^{\mathrm{M}_{\mathrm{i}}}$ that exhibits a distinct modulation with respect to the spatial coordinates $\vartheta$ and $\varphi$, as shown in Eq. (9). Using the metric coefficients given in Eq. (8) the original spherical wave function of the example $Y_{3,1}(\vartheta, \varphi)$, as depicted in Fig. 3, yields the set of modulated harmonics $Y_{3,1}^{\mathrm{M}_{\mathrm{i}}}(\vartheta, \varphi)$, as shown in Fig. 5. Equation (9) shows the new, functional dependence between Eq. (4) and the metric coefficients according to Eq. (8):

$$
\left\{\begin{array}{l}
Y_{\mathrm{n}, \mathrm{m}}^{\mathrm{e}, \mathrm{M}_{\mathrm{i}}} \\
Y_{\mathrm{n}, \mathrm{m}}^{\mathrm{o}, \mathrm{M}_{\mathrm{i}}}
\end{array}\right\}=M_{i}(\vartheta, \varphi) \cdot\left\{\begin{array}{l}
Y_{\mathrm{n}, \mathrm{m}}^{\mathrm{e}}(\vartheta, \varphi) \\
Y_{\mathrm{n}, \mathrm{m}}^{\mathrm{o}}(\vartheta, \varphi)
\end{array}\right\},
$$

with $i=1, \ldots, 4$.

In case of comparing different SME field approxmations by complex vector coefficients $\left(\underline{a}_{\mathrm{n}}^{\mathrm{m}}, \underline{b}_{\mathrm{n}}^{\mathrm{m}}\right)$, the equality of the underlying spherical harmonics between different field approaches is fundamental. Obviously, this is only realisable when the field approaches are based on the same spectra of spherical harmonics $Y_{\mathrm{n}, \mathrm{m}}(\vartheta, \varphi)$. Applying Eqs. (8) and (9) in order to map the surface current distribution to spherical coordinates and replacing the magnetic vector potential $\underline{\boldsymbol{A}}(\boldsymbol{r})$ in Eq. (7) by the expression given in Eq. (6) yields:

$$
\begin{aligned}
{\left[\begin{array}{l}
\underline{E}_{\vartheta} \\
\underline{E}_{\varphi}
\end{array}\right] } & =K(r) \sum_{n=0}^{\infty} \sum_{m=0}^{n} j^{\mathrm{n}} \cdot \\
& \left\{\left[\begin{array}{l}
\underline{a}_{\mathrm{x}}^{\mathrm{n}, \mathrm{m}} \cdot Y_{\mathrm{n}, \mathrm{m}}^{\mathrm{e}, \mathrm{M}_{1}}(\vartheta, \varphi)+\underline{a}_{\mathrm{y}}^{\mathrm{n}, \mathrm{m}} \cdot Y_{\mathrm{n}, \mathrm{m}}^{\mathrm{e}, \mathrm{M}_{2}}(\vartheta, \varphi) \\
\underline{a}_{\mathrm{x}}^{\mathrm{n}, \mathrm{m}} \cdot Y_{\mathrm{n}, \mathrm{m}}^{\mathrm{e}, \mathrm{M}_{3}}(\vartheta, \varphi)+\underline{a}_{\mathrm{y}}^{\mathrm{n}, \mathrm{m}} \cdot Y_{\mathrm{n}, \mathrm{m}}^{\mathrm{e}, \mathrm{M}_{4}}(\vartheta, \varphi)
\end{array}\right]\right. \\
+\quad & {\left.\left[\begin{array}{l}
\underline{b}_{\mathrm{x}}^{\mathrm{n}, \mathrm{m}} \cdot Y_{\mathrm{n}, \mathrm{m}}^{\mathrm{o}, \mathrm{M}_{1}}(\vartheta, \varphi)+\underline{b}_{\mathrm{y}}^{\mathrm{n}, \mathrm{m}} \cdot Y_{\mathrm{n}, \mathrm{m}}^{\mathrm{o}, \mathrm{M}_{2}}(\vartheta, \varphi) \\
\underline{b}_{\mathrm{x}}^{\mathrm{n}, \mathrm{m}} \cdot Y_{\mathrm{n}, \mathrm{m}}^{\mathrm{o}, \mathrm{M}_{3}}(\vartheta, \varphi)+\underline{b}_{\mathrm{y}}^{\mathrm{n}, \mathrm{m}} \cdot Y_{\mathrm{n}, \mathrm{m}}^{\mathrm{o}, \mathrm{M}_{4}}(\vartheta, \varphi)
\end{array}\right]\right\}, }
\end{aligned}
$$


Table 2. Coordinate plane dependency of the SME vector coefficient excitation

\begin{tabular}{|r|cc|cccc|ccccccc|}
\hline & $\mathbf{n}=\mathbf{0}$ & & $\mathbf{n}=\mathbf{1}$ & & & $\mathbf{n}=\mathbf{2}$ & & & & & \\
& $\underline{\boldsymbol{a}}_{0}^{0}$ & $\underline{\boldsymbol{b}}_{0}^{0}$ & $\underline{\boldsymbol{a}}_{1}^{0}$ & $\underline{\boldsymbol{b}}_{1}^{0}$ & $\underline{\boldsymbol{a}}_{1}^{1}$ & $\underline{\boldsymbol{b}}_{1}^{1}$ & $\underline{\boldsymbol{a}}_{2}^{0}$ & $\underline{\boldsymbol{b}}_{2}^{0}$ & $\underline{\boldsymbol{a}}_{2}^{1}$ & $\underline{\boldsymbol{b}}_{2}^{1}$ & $\underline{\boldsymbol{a}}_{2}^{2}$ & $\underline{\boldsymbol{b}}_{2}^{2}$ \\
\hline $\mathbf{x y}$ & $\mathrm{x}$ & - & - & - & $\mathrm{x}$ & $\mathrm{x}$ & $\mathrm{x}$ & - & - & - & $\mathrm{x}$ & $\mathrm{x}$ \\
\hline $\mathbf{x z}$ & $\mathrm{x}$ & - & $\mathrm{x}$ & - & $\mathrm{x}$ & - & $\mathrm{x}$ & - & $\mathrm{x}$ & - & $\mathrm{x}$ & - \\
\hline $\mathbf{y z}$ & $\mathrm{x}$ & - & $\mathrm{x}$ & - & - & $\mathrm{x}$ & $\mathrm{x}$ & - & - & $\mathrm{x}$ & $\mathrm{x}$ & $\mathrm{x}$ \\
\hline $\operatorname{index} i$ & 1 & 2 & 3 & 4 & 5 & 6 & 7 & 8 & 9 & 10 & 11 & 12 \\
\hline
\end{tabular}

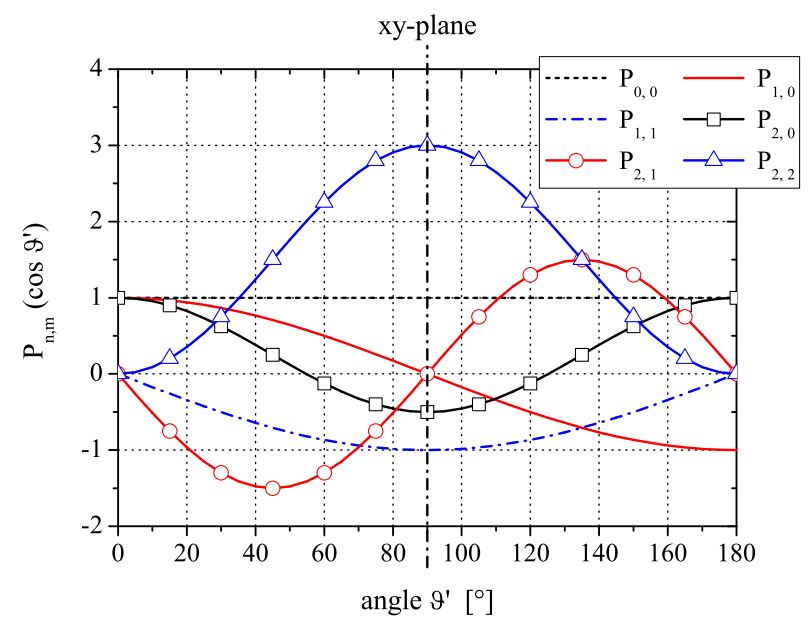

Fig. 6. Associated Legendre polynomials $P_{\mathrm{n}}^{\mathrm{m}}$ depending on the elevation angle $\vartheta^{\prime}$ of the underlying coordinate system.

where $K(r)$ merges the total radial functional dependency. In Eq. (6), $\underline{a}_{\mathrm{x}, \mathrm{y}}^{\mathrm{n}, \mathrm{m}}$ and $\underline{b}_{\mathrm{x}, \mathrm{y}}^{\mathrm{n}, \mathrm{m}}$ correspond to the vector expansion coefficients of the spherical field approach that can be directly computed from Eq. (11) using the surface current distribution on the antenna, as derived from the FEM solution.

Applying Fraunhofer relation, given by Eq. (7), to the electric far-fields, the obtained SME field approximation according to Eq. (10) is complete but its compactness is reduced due to the adaption of the spherical harmonics, which was inevitable in order to perform the requested coordinate transform. In detail, the next section focuses on the characterisation of antenna's surface currents in terms of complex modal vector expansion coefficients $\underline{\boldsymbol{a}}_{\mathrm{n}}^{\mathrm{m}}, \underline{\boldsymbol{b}}_{\mathrm{n}}^{\mathrm{m}}$.

\subsection{Modal characteristics of planar, log.-per. antennas}

In this section the representation of the antenna's surface current distribution $\underline{\boldsymbol{J}}_{s}$ in terms of SME vector coefficients $\underline{\boldsymbol{a}}_{\mathrm{n}}^{\mathrm{m}}$ and $\underline{b}_{\mathrm{n}}^{\mathrm{m}}$ will be extensively analysed by evaluating the following integral equation:

$$
\begin{aligned}
\left\{\begin{array}{l}
\left\{\underline{\boldsymbol{a}}_{\mathrm{n}}^{\mathrm{m}}\right. \\
\underline{\boldsymbol{b}}_{\mathrm{n}}^{\mathrm{m}}
\end{array}\right\}=\iint_{\left(A^{\prime}\right)} & N_{\mathrm{n}}^{\mathrm{m}} \cdot \underline{\boldsymbol{J}}_{s}\left(\boldsymbol{r}^{\prime}\right) \cdot j_{\mathrm{n}}\left(k r^{\prime}\right) \\
& \cdot P_{\mathrm{n}}^{\mathrm{m}}\left(\cos \vartheta^{\prime}\right) \cdot\left\{\begin{array}{c}
\cos \left(m \varphi^{\prime}\right) \\
\sin \left(m \varphi^{\prime}\right)
\end{array}\right\} d A^{\prime} .
\end{aligned}
$$

frequency $[\mathrm{GHz}]$

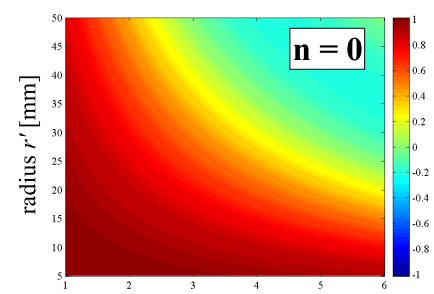

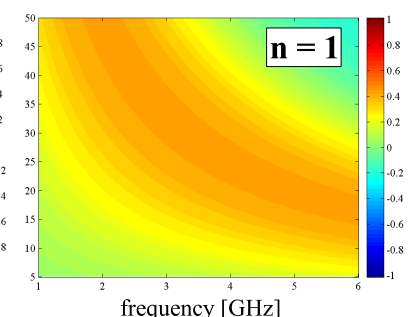

frequency $[\mathrm{GHz}]$
Fig. 7. Vector coefficient weighting characterized by spherical Bessel functions $j_{\mathrm{n}}\left(k r^{\prime}\right)$.

Besides the already established expressions in Eq. (11), $j_{\mathrm{n}}$ denotes a spherical Bessel function that has to be integrated across the entire planar antenna footprint. $j_{\mathrm{n}}$ performs a scaling of the surface current $\underline{\boldsymbol{J}}_{s}\left(\boldsymbol{r}^{\prime}\right)$ in dependence of its operational frequency and the position $\boldsymbol{r}^{\prime}$. Subsequently, the computation of the vector expansion coefficients $\underline{\boldsymbol{a}}_{\mathrm{n}}^{\mathrm{m}}$ and $\underline{\boldsymbol{b}}_{\mathrm{n}}^{\mathrm{m}}$ will be carried out for antenna elements located in parallel to the $x y$-plane. In this context the argument of the associated Legendre polynomials $P_{\mathrm{n}}^{\mathrm{m}}$ remains unchanged due to a constant elevation angle $\vartheta^{\prime}=0$ in the whole $x y$-plane.

In Fig. 6 the associated Legendre polynomials $P_{\mathrm{n}}^{\mathrm{m}}$ for different field expansion degrees and orders are depicted for the complete range of the elevation angle $\vartheta^{\prime}$. Nulls of discrete Legendre polynomials lead to a distinct fade-out of certain modal vector coefficients in the $x y$-plane in combination with the nulls of the trigonometric functions. Table 2 shows a complete set of vector expansion coefficients up to a maximum degree $n=2$ for surface current distributions limited to one of the three main planes of the cartesian coordinate system. Considering the symmetry relationship of the spherical eigenfunctions, the $x y$-plane might be the optimum choice with respect to an optimum far-field approximation.

Yet another modal characteristic is expressed by the spherical Bessel function $j_{\mathrm{n}}\left(k r^{\prime}\right)$, resulting in a radius- and frequency-dependent weighting factors. In Fig. 7 the amplitude scaling of the vector expansion coefficients $\underline{\boldsymbol{a}}_{\mathrm{n}}^{\mathrm{m}}$ and $\underline{\boldsymbol{b}}_{\mathrm{n}}^{\mathrm{m}}$ is analyzed with respect to a variable radius $r^{\prime}$ in an interval from 5 to $50 \mathrm{~mm}$ that covers the entire footprint of the antenna and the operational frequency range of the antennas from 1.0 to $6.0 \mathrm{GHz}$.

In Fig. 7 the spherical Bessel function $j_{\mathrm{n}}\left(k r^{\prime}\right)$ is evaluated 


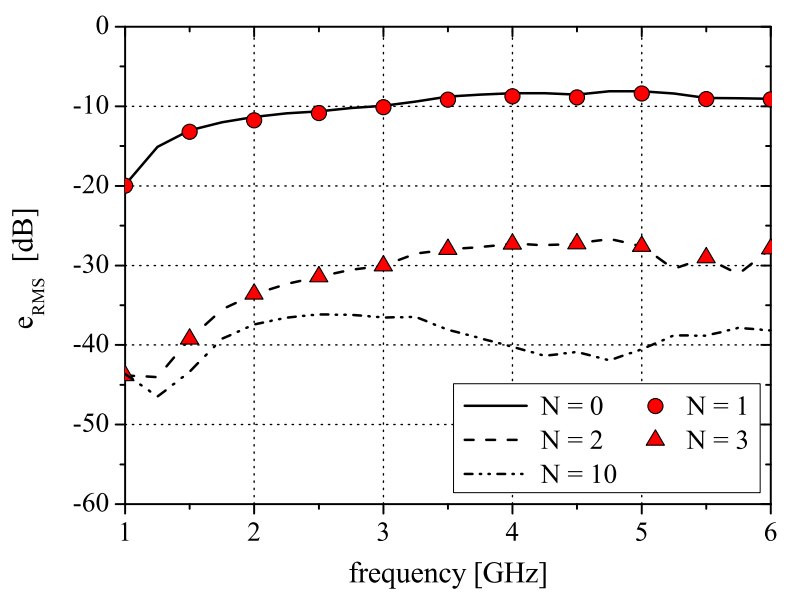

Fig. 8. RMS error in terms of different SME degrees $N$ for a trapezoidal antenna $(M=5)$.

for the first two consecutive SME degrees $n=0,1$ that illustrate the wavelike amplitude scaling of vector expansion coefficients with increasing radius of the antenna element and increasing frequency. Thus, the vector coefficient $\underline{\boldsymbol{a}}_{0}^{0}$ accounts for a maximum power allocation to the near-field sources close to the antenna center, whereas subsequent coefficients of the series expansion (as shown for SME degree $n=1$ ) emphasize regions that are aligned offset the center. As can be seen from Fig. 7, additional regions on the antenna surface exist, where the spherical Bessel function vanishes for a given frequency point. Therefore current sources for this given combination of location on the antenna surface and operational frequency do not contribute to the overall farfield radiation due to the fact that they are suppressed by the properties of the spherical Bessel function $j_{\mathrm{n}}\left(k r^{\prime}\right)$.

\section{Verification and convergence analysis}

In this section the verification of the spherical eigenfield approach is aspired and will be combined with an analysis of the convergence behaviour of the series expansion in terms of limiting the upper expansion degree to a finite value $N$. Only in this case a distinct numerical evaluation of the given SME approximation can be accomplished. The results derived by the SME expansion of the surface current distribution will be compared to the far-field (FF) results that were computed using a conventional moment-based calculation of Eq. (5).

According to Chen and Simpson (1991) the root-meansquare (RMS) error $e_{R M S}$ is used for the analysis in order to evaluate the exactness of the far-field quantities as derived from SME with the reference results. The RMS error computes the deviation between the far-field quantities for both electric field components $\boldsymbol{E}_{\vartheta}$ and $\boldsymbol{E}_{\varphi}$ in every requested far-

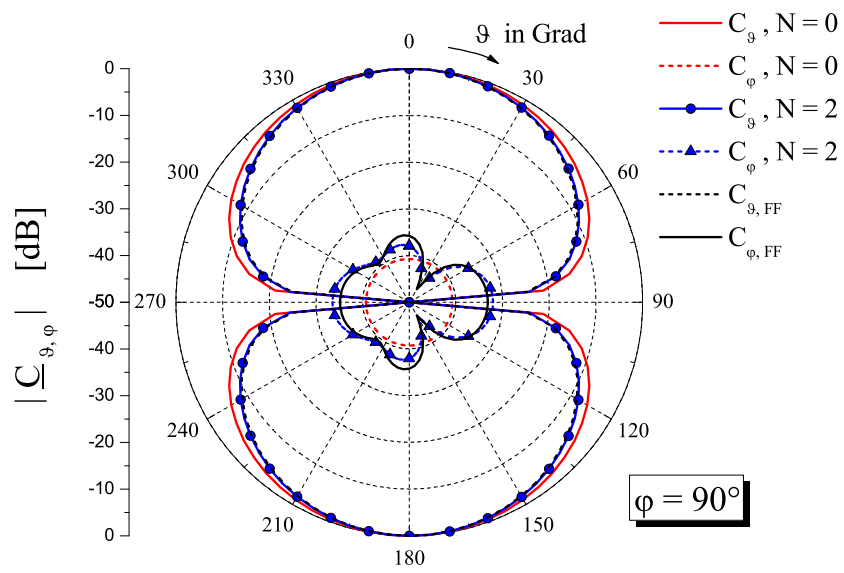

Fig. 9. Normalized radiation pattern $C_{\vartheta, \varphi}\left(\vartheta, \varphi=90^{\circ}\right)$ of a trapezoidal antenna $(M=5, f=2.5 \mathrm{GHz})$ in free space.

field point, as follows:

$$
\begin{aligned}
& e_{R M S}=\left[\frac{1}{K L} \sum_{k=1}^{K} \sum_{l=1}^{L}\{\right. \\
& \left(C_{\vartheta}^{\mathrm{ref}}\left(\vartheta_{k}, \varphi_{l}\right)-C_{\vartheta}^{\mathrm{comp}}\left(\vartheta_{k}, \varphi_{l}\right)\right)^{2} \\
& \left.\left.+\left(C_{\varphi}^{\text {ref }}\left(\vartheta_{k}, \varphi_{l}\right)-C_{\varphi}^{\text {comp }}\left(\vartheta_{k}, \varphi_{l}\right)\right)^{2}\right\}\right]^{1 / 2} \\
& \text { with } \quad C \underset{\vartheta, \varphi}{\text { ref,comp }}\left(\vartheta_{k}, \varphi_{l}\right)=\frac{\left|\underline{E}_{\vartheta, \varphi}^{\text {ref,comp }}\left(\vartheta_{k}, \varphi_{l}\right)\right|}{\left|\max \left\{\underline{E}_{\vartheta, \varphi}^{\text {ref,comp }}\right\}\right|} \text {, }
\end{aligned}
$$

where $C_{\vartheta, \varphi}^{\text {ref,comp }}$ denotes the normalized radiation characteristics in terms of the co- (CP) and cross-polarized (XP) components of the far-field of the related antenna element. In this context, the RMS error gives the total amount of field error in terms of one singular value regardless in which component or direction the field deviation occurs. In Fig. 8 the RMS error in $\mathrm{dB}$ is evaluated and depicted for a log.-per. four-arm antenna with $M=5$ periods of trapezoidal unit cell in the considered frequency range from 1.0 to $6.0 \mathrm{GHz}$ for different SME expansion degrees $N$.

As shown in Fig. 8, the spherical field approximation exhibits an erratic error behaviour with an increasing degree $N$ of the field expansion. Whereas the RMS error ranges above $-20 \mathrm{~dB}$ for expansion degrees $N=0,1$ the error is significantly reduced by applying expansion degrees $N=$ 2,3 and provides an already excellent far-field approximation with a marginal value of the RMS error ranging below $e_{R M S}<-25 \mathrm{~dB}$ over the entire frequency range of operation. In addition, increasing the maximum SME degree up to $N=10$ just offers a slight error improvement. Obviously, the discontinuities in the approximation depend on the discrete, modal excitation behaviour due to the vector coefficient's functional nulls, as exposed in Sect. 3.4. 


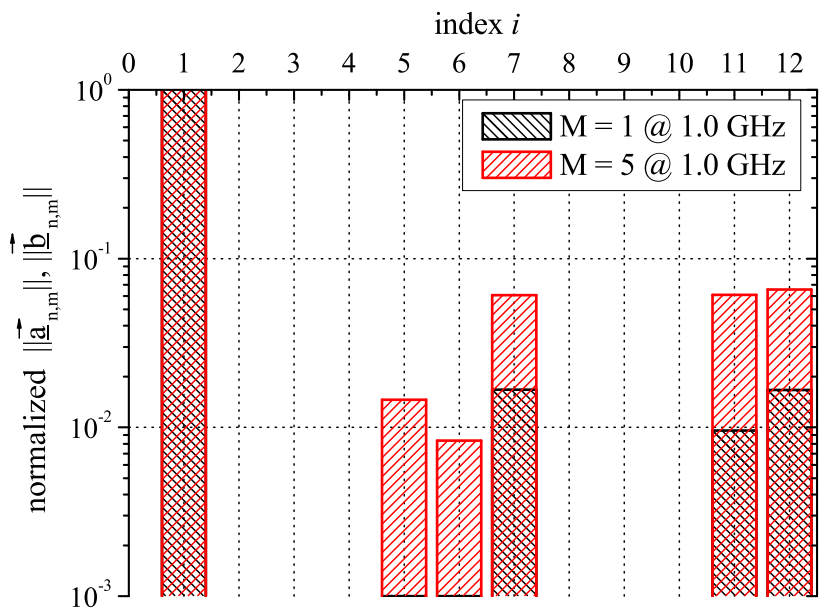

Fig. 10. Normalized vector coefficients at $f=1.0 \mathrm{GHz}$ for the antenna structures with $M=1.5$

Furthermore, in Fig. 9 the various radiation characteristics of the considered trapezoidal antenna are depicted, as utilized for the RMS error analysis that was shown in Fig. 8. The normalized antenna radiation patterns are depicted in the distinct far-field cutting plane of $\varphi=90^{\circ}$ at an operational frequency of $f=2.5 \mathrm{GHz}$ and separated with respect to its co- $(\vartheta-)$ and cross-polarized $(\varphi-)$ far-field components. This diagram directly highlights angular regions with a very high degree of field approximation and marks other regions that mainly contribute to an enhanced RMS error.

Figure 9 demonstrates the excellent copolar SME field approximation that is already obtained for very small degrees $N \geq 0$ in antenna main beam direction at $\vartheta=0^{\circ}$. Even the radiation pattern nulls at $\vartheta=90^{\circ}$ and $\vartheta=270^{\circ}$ are approximated almost perfectly. This is a remarkable property of the field expansion with modulated spherical harmonics as shown in Sect. 3.3 due to the fact that the original spherical harmonic $Y_{0,0}^{\mathrm{e}}(\vartheta, \varphi)$ represents a sphere in spherical coordinates $\vartheta$ and $\varphi$ and therefore provides no nulls at the angular positions $\vartheta=90^{\circ}$ and $\vartheta=270^{\circ}$. In contrast, the cross-polar field component $C_{\varphi}$ exhibits an attenuation by about $35 \mathrm{~dB}$ in antenna main beam direction and requires an SME field approximation of a higher degree. Therefore the cross polar component of the far-field exhibits a lower convergence behaviour than the co-polar component $C_{\vartheta}$. An improvement of the far-field approximation is obtained in terms of an increasing degree of the field expansion.

Recapitulating this Section, the chosen spherical currentbased eigenfield approach was verified and analyzed in terms of its field convergence behaviour, providing a basis for a significant parameter reduction with respect to the computation of antenna radiation fields and therefore may yield a much faster numerical evaluation.

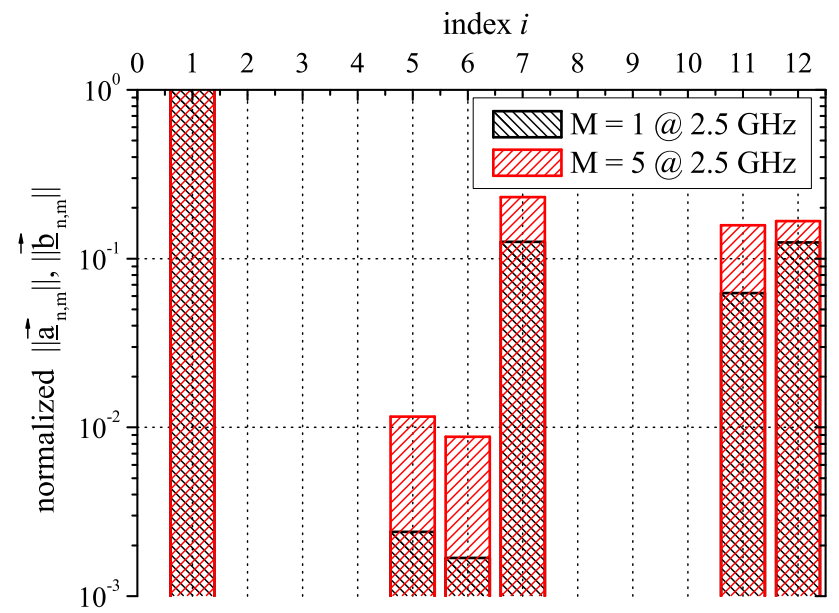

Fig. 11. Normalized vector coefficients at $f=2.5 \mathrm{GHz}$ for the antenna structures with $M=1,5$

\section{Modal four-arm trapezoidal antenna analysis}

As demonstrated in Sect. 3.4, the entire antenna's source region can be completely specified by vector coefficients of a spherical mode expansion. E.g. applying an approximation with a maximum degree $N=2$ of the field expansion incorporating $12 \mathrm{SME}$ vector coefficients yields an acceptable field approximation for both components of the far-field. Hence, the SME-based antenna analysis may be reduced to an in-depth consideration of the related modal SME vector coefficients as an equivalent source for far-field radiation.

For this purpose the extraction of dominant modal vector coefficients $\underline{\boldsymbol{a}}_{\mathrm{n}}^{\mathrm{m}}$ and $\underline{\boldsymbol{b}}_{\mathrm{n}}^{\mathrm{m}}$ will be identified and compared by means of the complex vector norm $\left\|\underline{\boldsymbol{a}}_{\mathrm{n}}^{\mathrm{m}}\right\|$ and $\left\|\underline{\boldsymbol{b}}_{\mathrm{n}}^{\mathrm{m}}\right\|$, as follows:

$$
\left\{\| \begin{array}{l}
\left\|\underline{\boldsymbol{a}}_{\mathrm{n}}^{\mathrm{m}}\right\| \\
\underline{\boldsymbol{b}}_{\mathrm{n}}^{\mathrm{m}} \|
\end{array}\right\}=\sqrt{\left\{\begin{array}{l}
\left|\underline{a}_{\mathrm{x}}^{\mathrm{nm}}\right|^{2} \\
\left|\underline{b}_{\mathrm{x}}^{\mathrm{nm}}\right|^{2}
\end{array}\right\}+\left\{\begin{array}{l}
\left|\underline{a}_{\mathrm{y}}^{\mathrm{nm}}\right|^{2} \\
\left|\underline{b}_{\mathrm{y}}^{\mathrm{nm}}\right|^{2}
\end{array}\right\} .}
$$

Furthermore, the complex vector norm given by Eq. (13) is normalized to the dominant coefficient, which is given by $\underline{\boldsymbol{a}}_{0}^{0}$. The real values are logarithmically scaled and depicted in Figs. 10 to 12 for three different exclusive frequencies of $1.0,2.5$ and $6.0 \mathrm{GHz}$, respectively. The analysis is conducted for two trapezoidal antennas with a variable number of basis function periods $M=1$ and $M=5$, as given in Sect. 2 . The abscissa scaling is given in terms of an index value $i$ according to Table 2 with its respective vector coefficients.

Besides the magnitude of the vector coefficient $\underline{a}_{0}^{0}$ (index 1) the evaluation of Figs. 10 to 12 points out three dominant coefficients, namely $\underline{\boldsymbol{a}}_{2}^{0}$ (index 7), $\underline{\boldsymbol{a}}_{2}^{2}$ (index 11) and $\underline{\boldsymbol{b}}_{2}^{2}$ (index 12). The magnitude of the other two excited coefficients $\underline{\boldsymbol{a}}_{1}^{1}$ (index 5) and $\underline{\boldsymbol{b}}_{1}^{1}$ (index 6) is significantly weaker with a level of about $-20 \mathrm{~dB}$ compared to the reference mode $\underline{\boldsymbol{a}}_{0}^{0}$. 


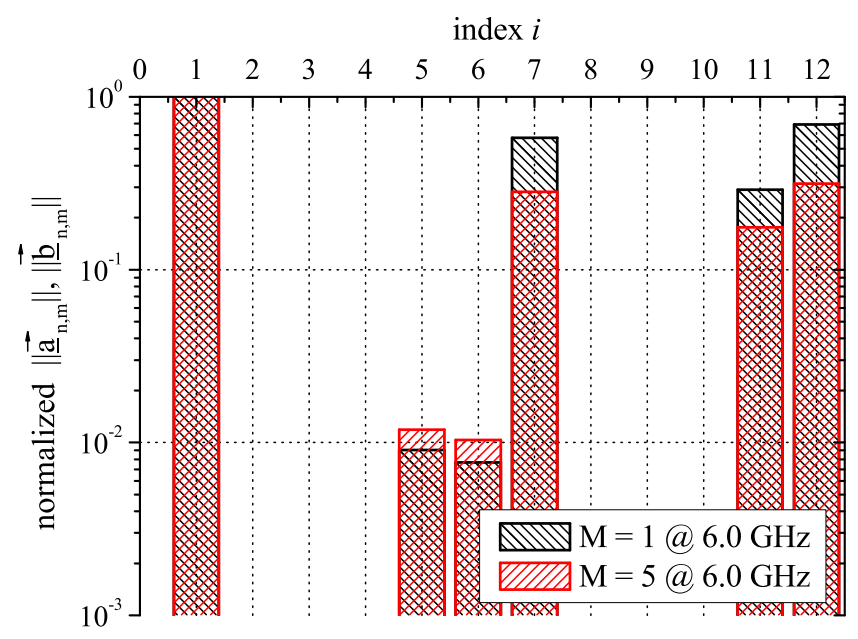

Fig. 12. Normalized vector coefficients at $f=6.0 \mathrm{GHz}$ for the antenna structures with $M=1,5$

From $f=1.0 \mathrm{GHz}$ depicted in Fig. 10 to $f=6.0 \mathrm{GHz}$ shown in Fig. 12 the modal vector differences between both trapezoidal antennas are noticeable decreasing. The variation especially at $f=1.0 \mathrm{GHz}$ between the two vector magnitudes is related to the reflections at the end of the arms. Otherwise, at $f=6.0 \mathrm{GHz}$ both antenna geometries operate in their frequency-independent radiation behaviour which can be seen from the almost identical magnitudes of the normalized vector coefficients (see Fig. 12). In Fig. 11 at $f=2.5 \mathrm{GHz}$ the structure with $M=5$ periods radiates in a frequency-independent travelling wave mode, whereas the second structure with $M=1$ periods of the trapezoidal unit cell is still predominated by resonant radiation characteristics.

As shown in this section, the consideration and the analysis of four dominant current-based vector quantities allows a deeper insight into the radiation behaviour of planar, trapezoidal antennas, combined with a faster numerical far-field antenna analysis due to a significant parameter reduction compared to conventional methods.

\section{Conclusions}

In this paper, planar frequency-independent log.-per. trapezoidal antenna structures are analysed by means of surface current distribution and a spherical eigenfield approach. Antennas of that kind are characterized by a high impedance bandwidth combined with a dual linear-polarized radiation behaviour which can be favorably used in future MIMOand multimode diversity communication systems. The generalized spherical mode expansion was adapted and verified by different convergence criteria with respect to fieldquantities as derived from conventional full-wave analysis applying the FEM. Leading to a significant parameter re- duction and a complete coordinate separation between the sources in the near-field and the requested far-field region, a modified spherical wave expansion was accomplished that yielded a description of antenna radiation fields by means of a distinct and solely antenna-dependent set of complex expansion coefficients. The radiation behaviour analysis of two different trapezoidal antenna structures was accomplished by comparing the two distinct sets of modal expansion coefficients that were directly related to the operational mode of the antenna, in order to highlight the intermediate relationship between the set of vector expansion coefficients and the shape of the antenna, respectively. Furthermore, in Klemp et al. (2006) this kind of current-based SME technique is utilized to deduce equations for a modal antenna correlation analysis in terms of MIMO transmission schemes using trapezoidal planar antennas with dual linear excitation.

\section{References}

Bruning, J. H. and Lo, Y. T.: Multiple Scattering of EM Waves by Spheres Part I-Multipole Expansion and Ray-Optical Solutions, IEEE Transactions on Antennas and Propagation, 19, 378-390, 1971.

Chen, Y. and Simpson, T.: Radiation Pattern Analysis of Arbitrary Wire Antennas Using Spherical Mode Expansions with Vector Coefficients, IEEE Transactions on Antennas and Propagation, 39, 1716-1721, 1991.

Chen, Y., Simpson, T. L., and Ho, T. Q.: Highly efficient technique for solving radiation and scattering problems, IEE Proc.-H, 139, 7-10, 1992.

Jensen, M. A. and Wallace, J. W.: A Review of Antennas and Propagation in MIMO Wireless Communications, 52, 2810-2824, 2004.

Klemp, O. and Eul, H.: Radiation Pattern Analysis of Antenna Systems for MIMO and Diversity Configurations, Adv. Radio Scie., 3, 157-165, 2005.

Klemp, O., Schultz, M., and Eul, H.: Novel logarithmically periodic planar antennas for broadband polarization diversity reception, Int. J. Electron. Commun. (AEUE), 59, 268-277, 2005.

Klemp, O., Armbrecht, G., and Eul, H.: Computation of Antenna Pattern Correlation and MIMO Performance by means of Surface Current Distribution and Spherical Wave Theory, Accepted for publication in Adv. Radio Scie., 2006.

Ludwig, A. C.: Near-Field Far-Field Transformations Using Spherical-Wave Expansions, IEEE Transactions on Antennas and Propagation, 19, 214-220, 1971.

Narasimhan, M. S., Christopher, S., and Varadarangan, K.: Modal Behavior of Spherical Waves from a Source of EM Radiation with Application to Spherical Scanning, 33, 350-354, 1985.

Potter, P. D.: Application of Spherical Wave Therory to Cassegrainian-Fed Paraboloids, IEEE Transactions on Antennas and Propagation, 15, 727-736, 1967.

Stratton, J. A.: Electromagnetic Theory, McGraw Hill, New York, 1941.

Werner, D. H. and Mittra, R.: Frontiers in Electromagnetics, IEEE Press Series on Microwave Technology and RF, New York, 2000. 